\title{
Fabrication of high quality optical coherence tomography (OCT) calibration artefacts using femtosecond inscription.
}

\author{
Graham C. B. Lee*a, Janarthanan Rasakanthan ${ }^{\mathrm{a}}$, Peter D Woolliams ${ }^{\mathrm{b}}$, Kate Sugden ${ }^{\mathrm{a}}$

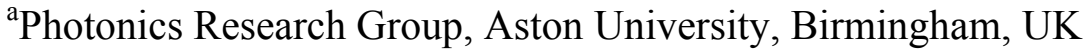 \\ ${ }^{b}$ Nano \& Functional Materials Group, National Physical Laboratory, Hampton Rd, Teddington, UK
}

\begin{abstract}
Optical coherence tomography (OCT) is a non-invasive three-dimensional imaging system that is capable of producing high resolution in-vivo images. OCT is approved for use in clinical trials in Japan, USA and Europe. For OCT to be used effectively in a clinical diagnosis, a method of standardisation is required to assess the performance across different systems. This standardisation can be implemented using highly accurate and reproducible artefacts for calibration at both installation and throughout the lifetime of a system. Femtosecond lasers can write highly reproducible and highly localised micro-structured calibration artefacts within a transparent media. We report on the fabrication of high quality OCT calibration artefacts in fused silica using a femtosecond laser. The calibration artefacts were written in fused silica due to its high purity and ability to withstand high energy femtosecond pulses. An Amplitude Systemes s-Pulse Yb:YAG femtosecond laser with an operating wavelength of $1026 \mathrm{~nm}$ was used to inscribe three dimensional patterns within the highly optically transmissive substrate. Four unique artefacts have been designed to measure a wide variety of parameters, including the points spread function (PSF), modulation transfer function (MTF), sensitivity, distortion and resolution - key parameters which define the performance of the OCT. The calibration artefacts have been characterised using an optical microscope and tested on a swept source OCT. The results demonstrate that the femtosecond laser inscribed artefacts have the potential of quantitatively and qualitatively validating the performance of any OCT system.
\end{abstract}

Keywords: Optical coherence tomography, femtosecond laser inscription, point spread function, sensitivity, distortion, standardisation, characterisation.

\section{INTRODUCTION}

Optical coherence tomography (OCT) is a three dimensional non-invasive imaging methodology $[1,2]$. There is a growing need to be able to characterise and calibrate OCT systems. This becomes more apparent as OCT systems become more widespread in medical fields as well as others. For OCT to continue to grow and develop, it is essential to have a method and phantoms (artefacts) that can be used as a benchmark to characterise and compare the performance of one system to another. Performance indicators such as the point spread function (PSF), modulation transfer function (MTF), sensitivity, distortion and resolution are all important. Lack of standardisation of OCT measurements will lead to uncertainty of a measurement result. For a standard to be established, an artefact would have to be easy to use, easily reproducible and be relatively low cost. In addition, it would be ideal if one artefact can be used to perform major and routine calibrations for more than one OCT performance indicator.

Previously, Tomlins et al [3] and Agrawal et al [4] used phantoms made by embedding micro particles in polyurethane resin (solid) and suspended them in aqua solution. These phantoms have been used to measure axial and lateral resolutions, MTF and contrast of the OCT systems [3-6]. The particles in these phantoms were randomly distributed, and they are limited to measuring the resolution of the system. Tomlin et al have demonstrated the potential of using femtosecond inscription for producing well-structured phantoms [7]. Later, a detailed parametric study of femtosecond laser inscription was conducted by Rasakanthan et al to evaluate the suitable inscription parameters [8]. Curatolo et al have also demonstrated fabrication of a structured phantom [9] .

*leegcb@aston.ac.uk; phone +44 121204 3498; fax +44 1212043682; www.aston.ac.uk

Biophotonics: Photonic Solutions for Better Health Care III, edited by Jürgen Popp,

Wolfgang Drexler, Valery V. Tuchin, Dennis L. Matthews, Proc. of SPIE Vol. 8427, 84271K

(C) 2012 SPIE $\cdot$ CCC code: $1605-7422 / 12 / \$ 18 \cdot$ doi: $10.1117 / 12.922348$

Proc. of SPIE Vol. $842784271 \mathrm{~K}-1$ 
With the advent of the femtosecond laser (fs) it is now possible to directly perform high-resolution micromachining and inscription in a wide range of planar and fibre devices [10]. Fs laser inscription has been applied to some tough materials such as diamond [11], ceramic [12] and silica [13]. The interaction between the ultrashort laser pulse and a material is largely due to a nonlinear process whereby multi-photon absorption is dominant [10]. With a suitable numerical aperture machining lens, machining translation speed and energy per pulse it is possible to create extremely localised changes around the laser focal spot within a material [14]. In addition, with a sample mounted on a multi-axis stage it is possible to create complex three dimensional structures inside a transparent material.

This paper demonstrates the fabrication of phantoms using a femtosecond (fs) laser to inscribe a variety of geometrical structures within the bulk of fused silica. These structures have been characterised using an optical microscope and a swept-source OCT system. Quantitative methods are under development that use the data obtained using these structures to quantitatively evaluate the performance parameters such as resolution, resolution degradation, MTF, sensitivity fall off and distortion of an OCT system. In addition, the same artefact can be used to recalibrate an OCT system. The highly reproducible and the deterministic nature of fs laser inscription process enable rapid production of high quality artefacts.

\section{EXPERIMENTAL SETUP}

The layout of the fs laser writing setup is illustrated in Figure 1. Laser pulses are generated by a mode-locked Yb:YAG fs laser (Amplitude Systemes s-Pulse HP) with a centre wavelength of $1026 \mathrm{~nm}$ with an average pulse duration below $500 \mathrm{fs}$. The laser has a tuneable pulse repetition rate between 1-100 kHz, all inscriptions in this study were conducted at $100 \mathrm{kHz}$ repetition. A FWHM laser spot size focused by the 100x (Mititoyo M Plan Apo NIR Series) objective with 0.5 NA was approximately $1.5 \mu \mathrm{m}$. The fused silica sample was cleaned using methanol and mounted on to goniometers to allow for tilt correction during the alignment of the sample. The goniometers, with the sample, were secured on to a subnanometre precision XY air-bearing translation stage with a mechanical Z translation (Aerotech ABL1000). A second common mechanical $\mathrm{Z}$ translation stage was used to control the focus of the machining lens and off axis camera. All stages were controlled through a control unit (Aerotech A3200) connected to a PC. The motion of the translation stages were programmed using G-Code commands for high precision stage movements. A fused silica sample was initially roughly aligned so that the surface of the sample was perpendicular to the machining lens. A series of lines were written across the surface of the sample at different heights. Using the off axis camera system it was then possible to establish which machining height produced the line that was at the surface of the sample. The series of lines were also used to correct for tilt through adjusting the goniometers. Once the surface of the sample was aligned perpendicular to the machining lens, a G-Code program was used to translate the stages at constant velocities so that the fs laser interacted with the sample to fabricate the intended structures.

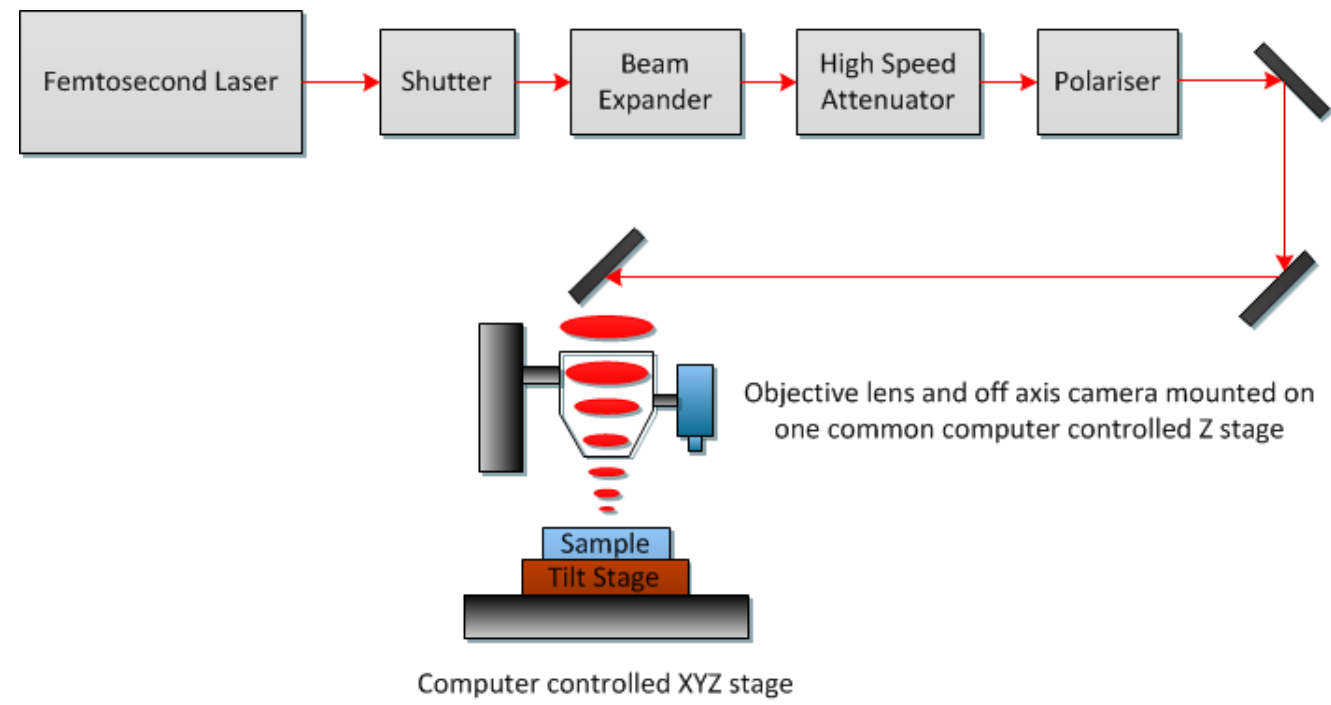

Figure 1. Schematic of the femtosecond laser setup. 


\section{PHANTOM DESIGN}

All designs have eight layers; the top layer was inscribed $100 \mu \mathrm{m}$ below the surface of the sample. Layers on PSF and sensitivity artefacts were offset in order to reduce the back scattering interference from other layers during OCT measurements. Subsequent layers were inscribed with a relative offset in the Y direction of $50 \mu \mathrm{m}$ at a translation speed of $10 \mathrm{~mm} / \mathrm{s}$. In this work, the PSF, departing and grid structures were inscribed at $860 \mathrm{~nJ}$ per pulse, to ensure the structures were visible when imaged by the majority of OCT systems. Whilst the sensitivity structure was written using $380 \mathrm{~nJ}$ to $1.3 \mathrm{~mJ}$ per pulse.

\subsection{PSF and Sensitivity artefact design:}

Figure 2 shows the designs of the PSF and sensitivity artefact. The PSF phantom contains 110 lines on each layer, with a line spacing of $150 \mu \mathrm{m}$ and a layer offset of $25 \mu \mathrm{m}$. The major difference between the sensitivity and PSF artefacts are that all the structures in PSF were inscribed with the same energy and at constant speed, whereas those in the sensitivity were inscribed with varying energy and at a constant speed.

The sensitivity phantom contains six sets of lines in the XY plane, each with five pairs of lines, separated by index lines. The index lines are used as a position reference i.e. set $\mathrm{n}$ is inscribed between $\mathrm{n}$ and $\mathrm{n}+1$ index lines. The energy of each pair is varied, a total of 30 pairs of lines with 30 different energies were used. The amount of back scattered light is proportional to the refractive index and the area of the scattering. The structures in the sensitivity artefact were fabricated with varying index and size; therefore, these can be used to identify the sensitivity limit of the system. The sensitivity of the system is defined as the reciprocal of the minimum reflection. The smallest feature that the OCT system can resolve will determine the sensitivity.

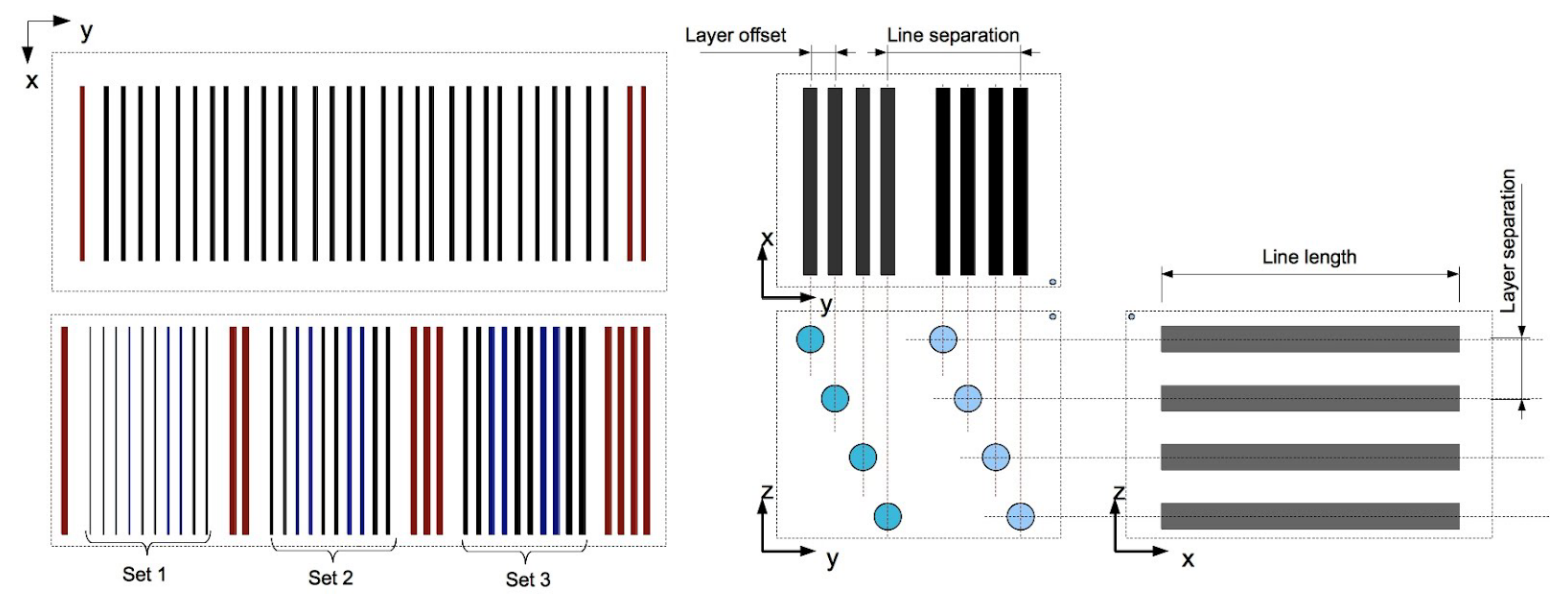

Figure 2. Schematic of the a) PSF design (top view) and b) sensitivity design (top view). c) Detailed view of a section of the PSF and sensitivity design. Image source [8].

\subsection{Departing Lines and Grid}

Figure 3 shows the design of one of the layers in the departing lines and grid artefact. The departing lines artefact was designed so that the spacing between the lines increased linearly - varied from 1 to $110 \mu \mathrm{m}$. Lines were separated into sets; each set had 10 spacings (i.e. 11 lines) and index lines to separate each set. All layers were inscribed without a layer-offset.

Distortion of an OCT system can arise from optical aberration and scanning methods/geometric. Theoretical models have been developed over the years to compensate for these distortions $[15,16]$. Development of a suitable phantom for quantification of three dimensional distortion is essential. We have created a three-dimensional grid using fs laser inscription. In the results presented here, grids with dimensions $100 \mu m \times 100 \mu m$ were inscribed. The distortion of the 
OCT can be deduced by measuring the deviation of the intersecting point from the ideal location. Using this information the distortion can then be corrected.

a)

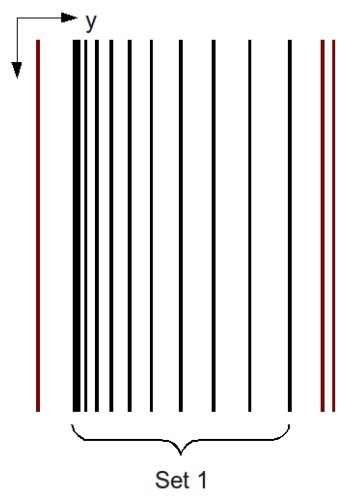

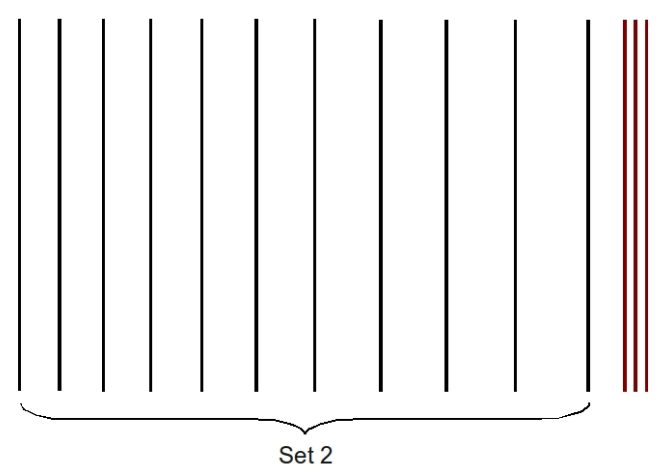

b)

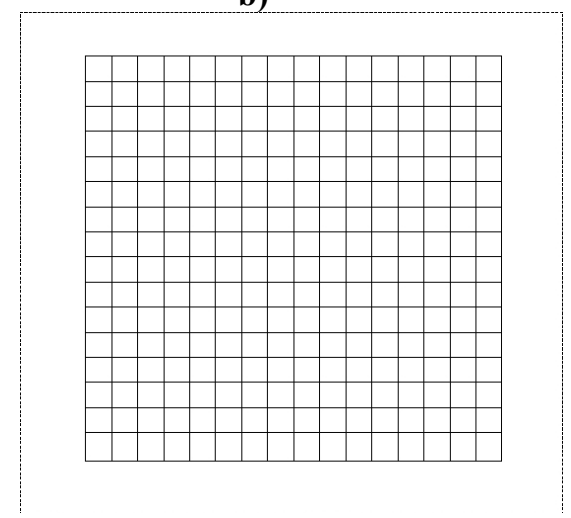

Figure 3. Schematic top down view of the a) departing lines pattern and b) the grid pattern.

\section{RESULTS AND DISCUSSION}

All the designs were characterised using an optical microscope (Axioscope-2 MOT plus, Zeiss) and a swept-source OCT (EX1301, Michelson Diagnostic Ltd, UK) operating at a central wavelength of $1305 \mathrm{~nm}$. This OCT system has an axial and lateral resolution of $10.9 \pm 0.2 \mu \mathrm{m}$ and $8.4 \pm 0.2 \mu \mathrm{m}$ respectively [6]. The OCT results are presented in pixel scale, where one pixel is approximately equal to $4 \mu \mathrm{m}$ in all axes. The sample was mounted with a small tilt to avoid the strong back reflections hindering the internal structures.

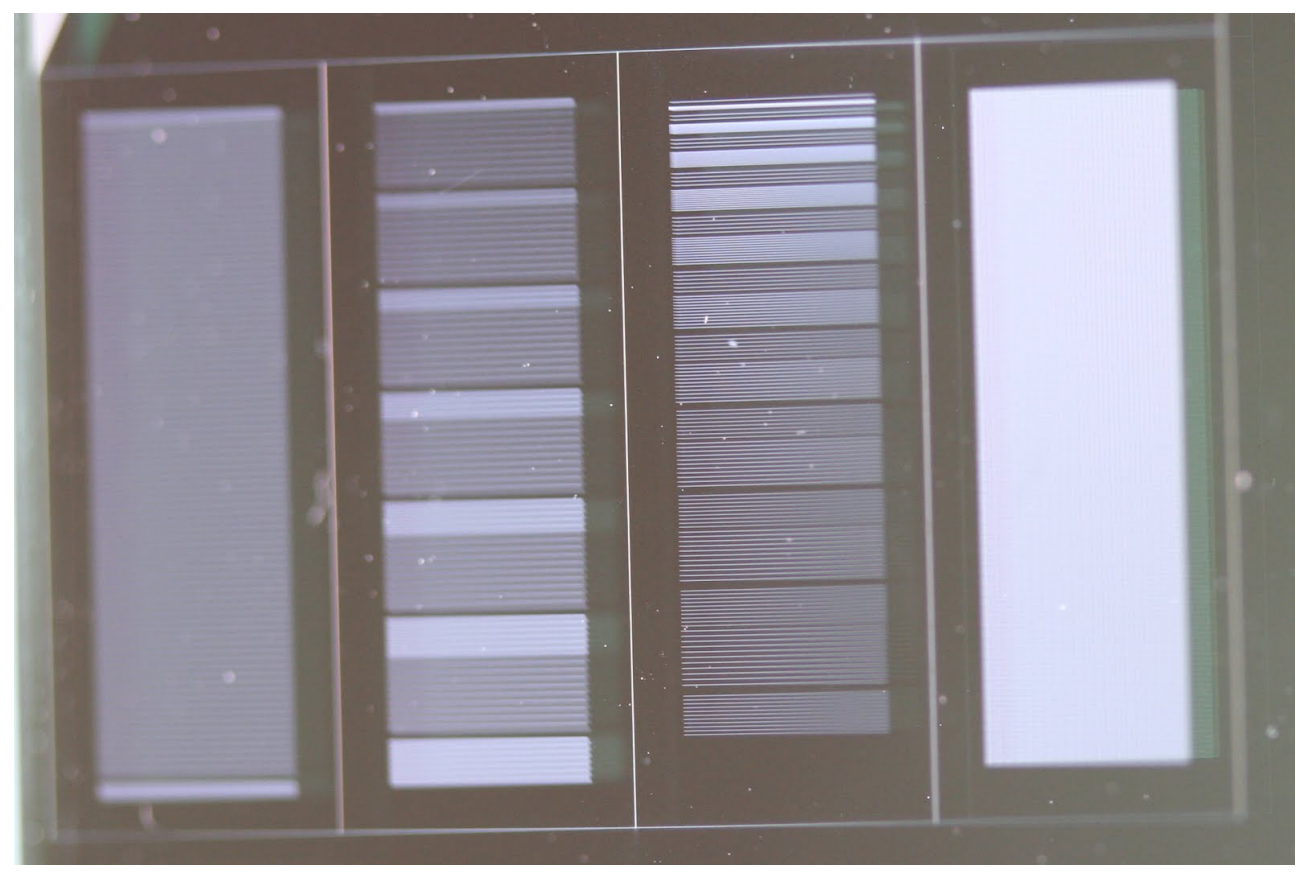

Figure 4. Photograph of the four artefacts written in a fused silica sample. (From left to right) PSF, sensitivity, departing and grid artefacts. 


\subsection{PSF and Sensitivity:}

When the cross section of the structures are smaller than the resolution of an OCT system, the observed results can be approximated to the two-dimensional PSF of the system. Hence direct measurement of the PSF is possible. Also, the modulation transfer function (MTF) or contrast transfer function (CTF) of the system can be calculated by measuring the line spread function (LSF) in the axial and lateral direction and then taking Fourier transform of these. Additionally, these phantoms can be used to measure the spatial variation of the resolution (i.e. variation of resolution with axial and lateral positions) sensitivity, signal drop off as well as to verify the linearity of the scan. The microscope images in Figure 5 illustrates the quality of the inscribed lines in both the PSF and sensitivity patterns.

a

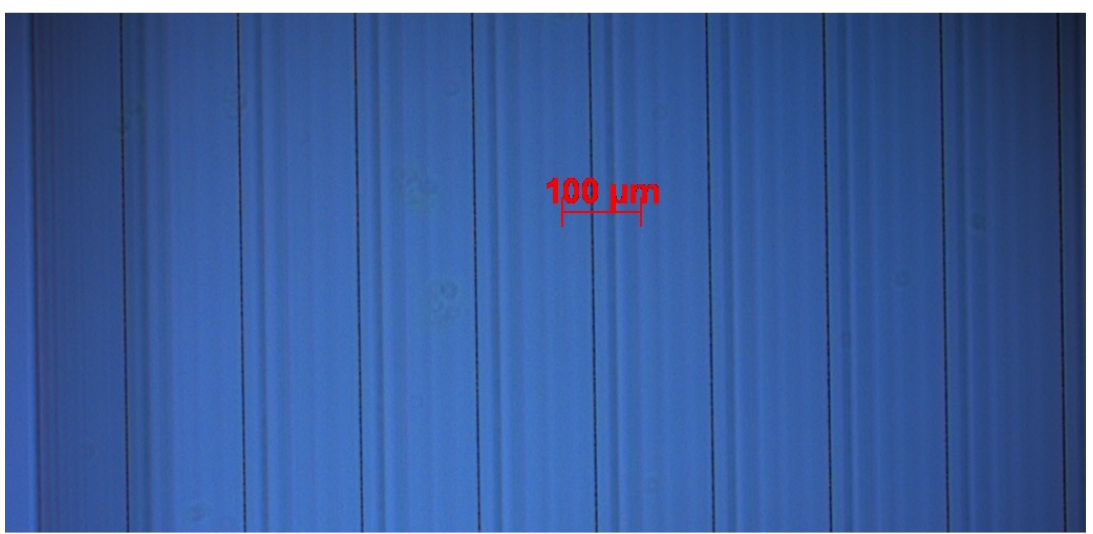

b

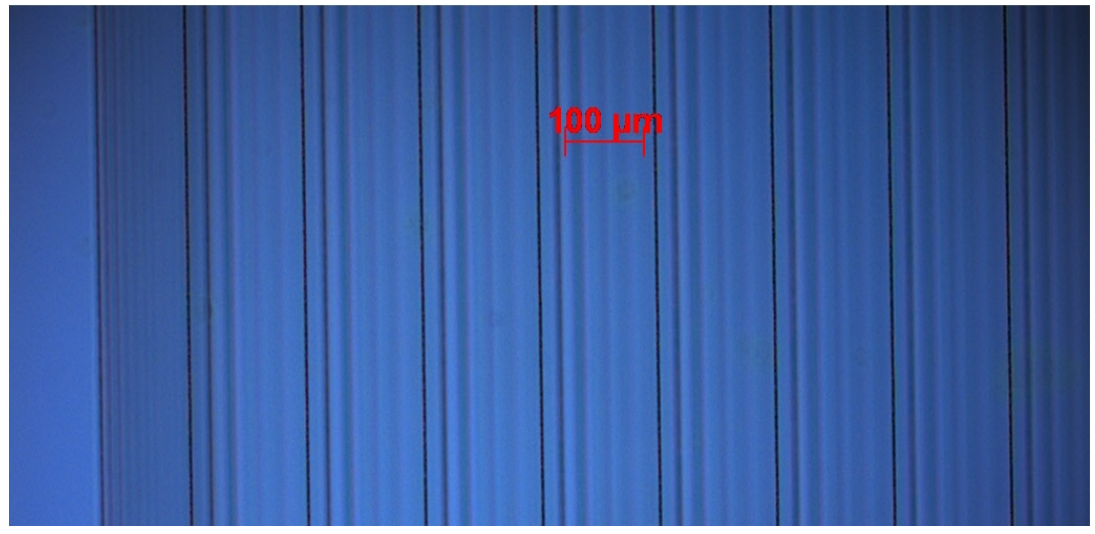

Figure 5. Microscope images showing the top layer of a) PSF and b) Sensitivity artefacts obtained using 5X objective.

Figure 6 shows part of a B-scan image of the PSF phantom, and the en-face image of the first layer. Spatial variant PSF and MTF can be computed around each of the detected points. For example, whilst the structures are smaller than the resolution of the system being investigated, a two-dimensional Gaussian function can be fitted to estimate the axial and lateral resolution. A spatial variant PSF map can be created by measuring the PSF around each point; this map can then be used for spatially variant deconvolution to improve resolution. In addition, total reflection from all the structures are approximately identical, therefore, the relative sensitivity/signal roll off of an OCT system can be deduced by measuring the integrated intensity. 

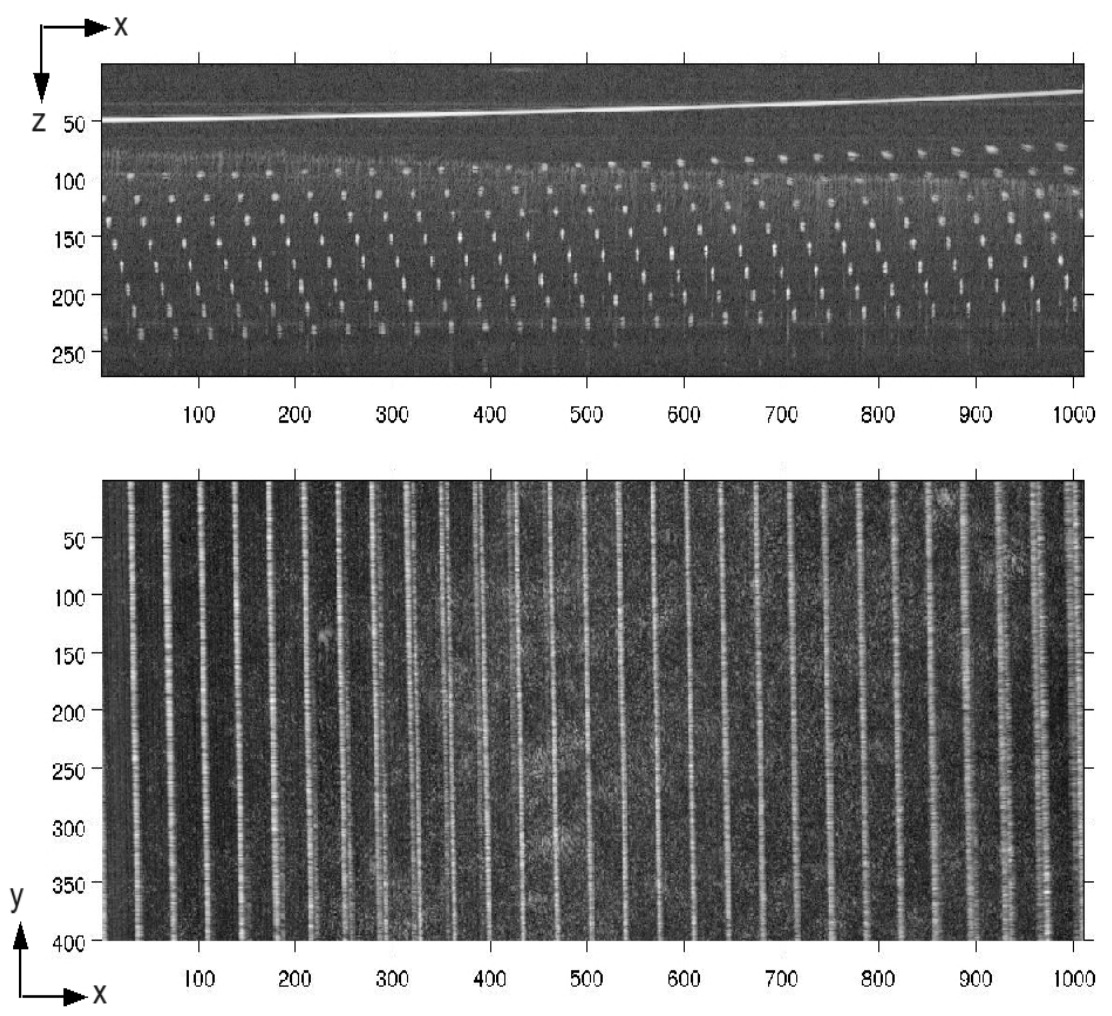

Figure 6. OCT image a) B-scan and b) en-face view of the 'PSF', where one pixel equates to approximately $4 \mu m$ along all axes.

Figure 7 shows the B-scan of set one from the sensitivity phantom and the en-face image of the first layer separated by the index lines. The PSF phantom can be used to measure the spatial variation of the sensitivity as well as the signal drop-off.

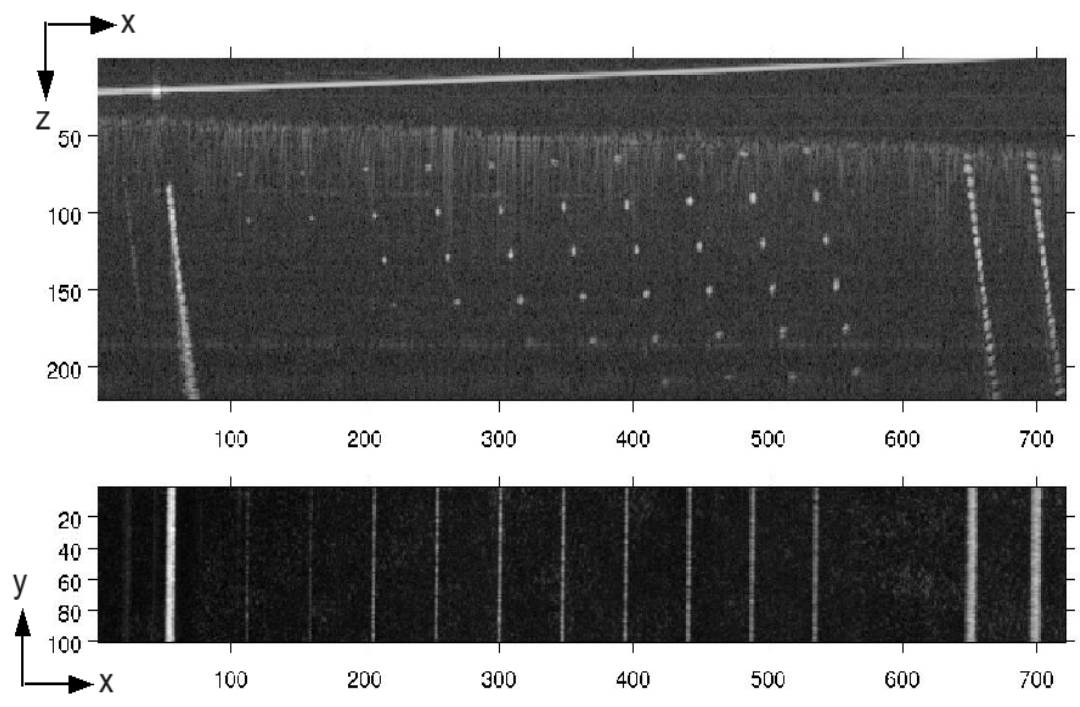

Figure 7. OCT image a) B-scan and b) en-face view of the sensitivity artefact. 


\subsection{Departing Lines}

The departing lines phantom was designed to enable a quick qualitative check of the resolution of an OCT system. The resolution limit of the system is determined by the smallest spacing the system can resolve, which can be related to the Rayleigh criterion for resolution. Figure 8a shows the microscope images of the first set spacing between 1-10 $\mu \mathrm{m}$ taken using $63 \mathrm{X}$ objective. Figure $8 \mathrm{~b}-\mathrm{c}$ shows set two and three with a line spacing of 11-20 and 21-30 $\mu \mathrm{m}$ respectively, obtained using $20 \mathrm{X}$ objective. The lateral width of the lines is $4.8 \mu \mathrm{m}$, therefore, a spacing smaller than $4.8 \mu \mathrm{m}$ is not physically resolvable as shown in Figure 8a.

a)

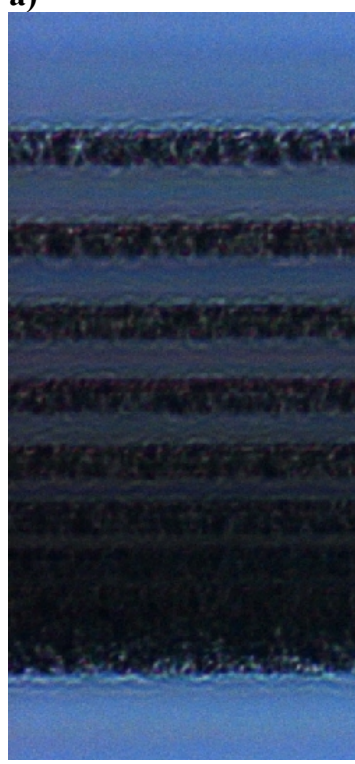

b)

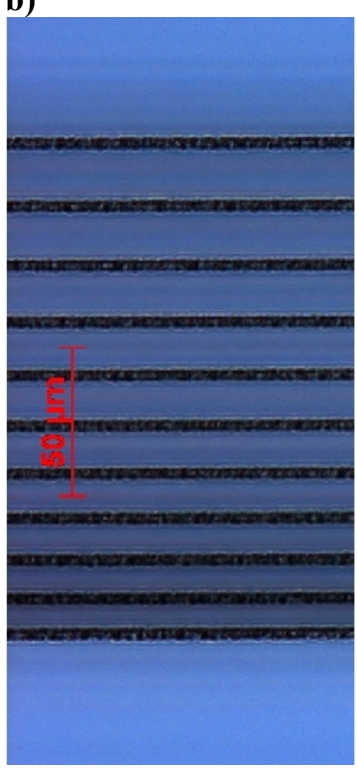

c)

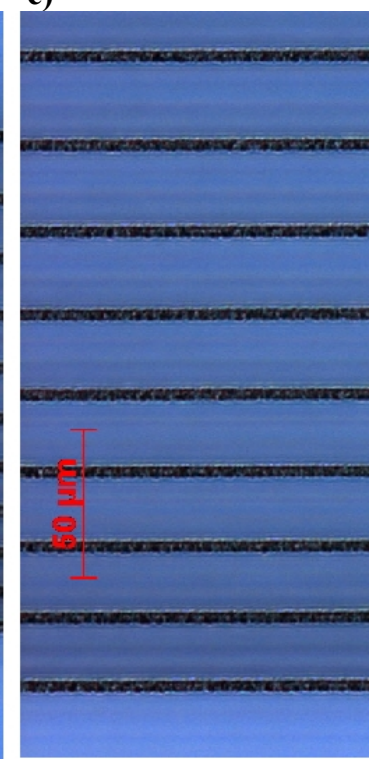

Figure 8. Microscope images of the sensitivity phantom a) set 1, spacing 1-10 $\mu$ m, taken with $63 x$ objective, b-c) set 2-3, spacing 11-20 and 21-30 $\mu \mathrm{m}$ respectively, taken with $20 \mathrm{x}$.

Figure 9a shows the B-scan image of set 1 to 5 (i.e. lines spacing 1-50) of the sensitivity phantom, where each set is separated by index lines. Figure $9 \mathrm{~b}$ shows the en-face image of the first layer. From this result, it is clear that individual points are resolvable between line spacing 8-9, which is comparable to the resolution of the OCT system used.

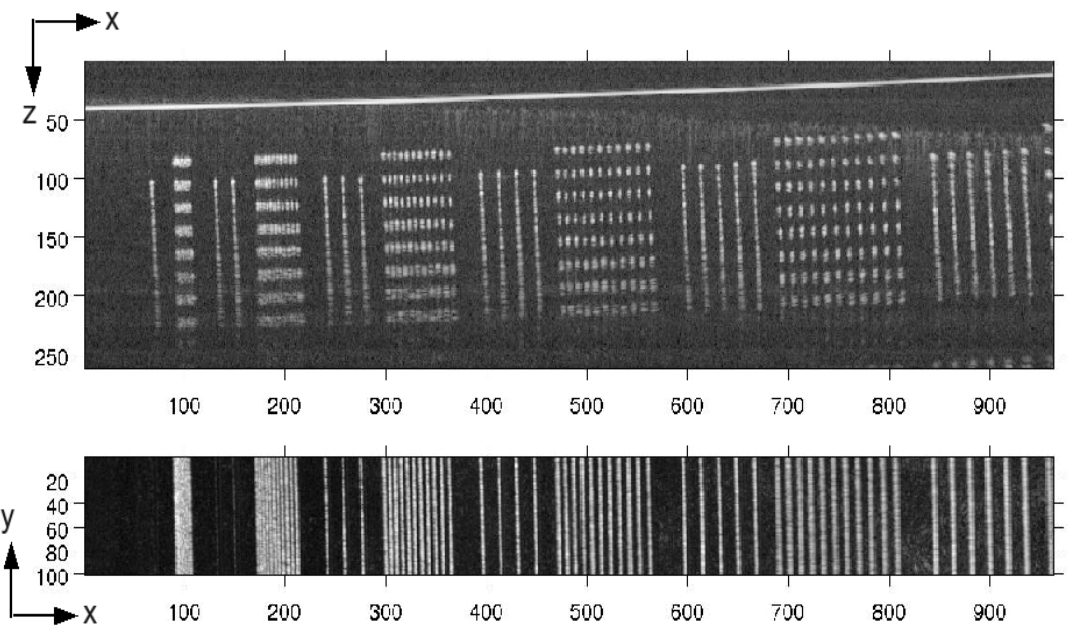

Figure 9. OCT image a) B-scan and b) en-face view of the departing lines. 


\subsection{Grid}

The grid phantoms was designed to measure the three dimensional distortion of the OCT system. In addition, this can be used to measure other matrices such as resolution, and spatially variant resolution and sensitivity as well. Figure 10 shows the microscope image of the first layer and Figure 11 shows the B-scan and en-face (top layer) of the phantom. Distortion of the system can be measured by identifying the deviation of the intersections from the ideal image.

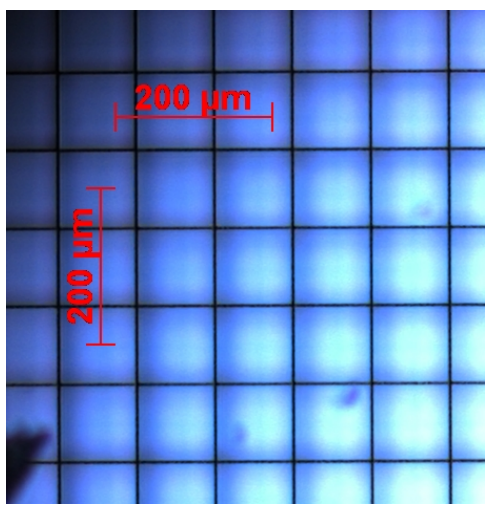

Figure 10. Microscope image of the grid top layer (100 $\mu \mathrm{m}$ bellow front surface) obtained with 20X objective.
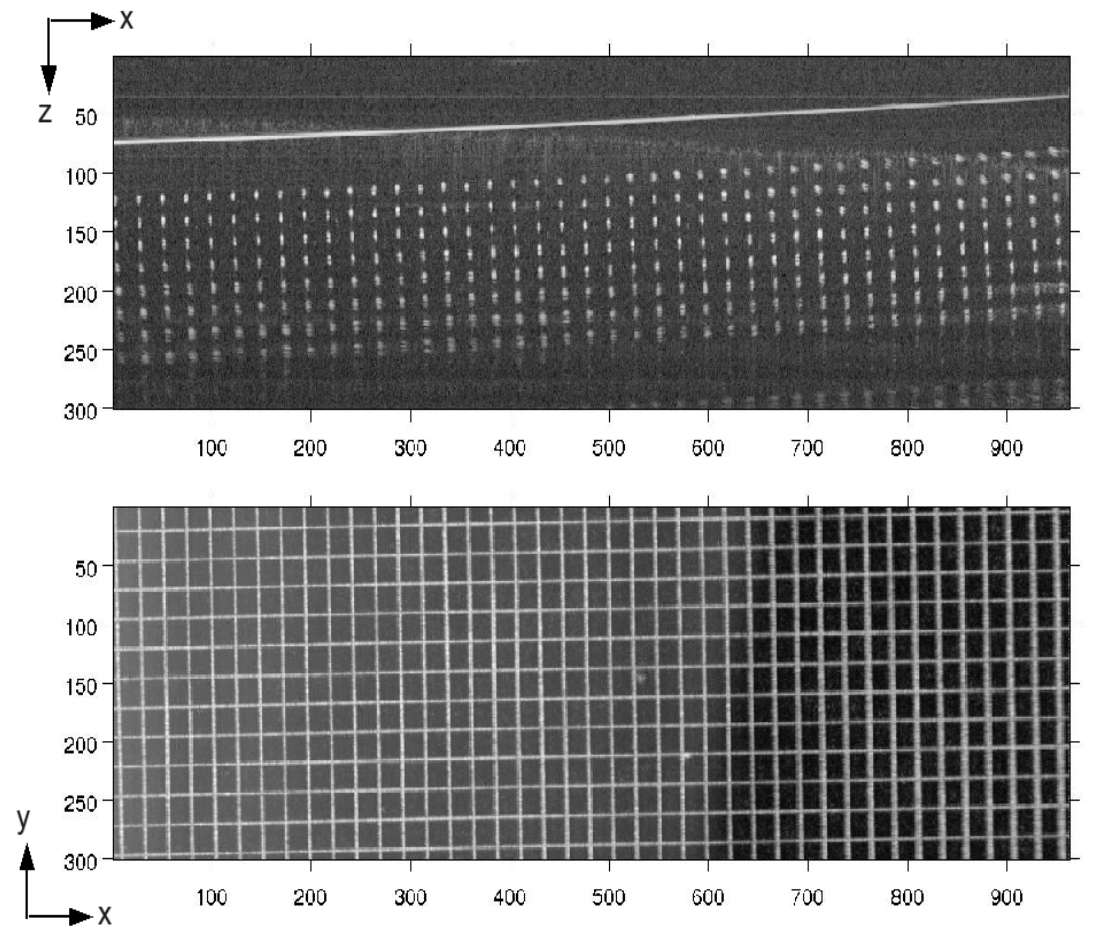

Figure 11. OCT image a) B-scan and b) en-face view of the grid artefact. 


\section{CONCLUSION}

Highly reproducible and durable OCT calibration artefacts were fabricated using femtosecond laser inscription. Phantoms were designed for quantitative calibration and characterisation of any OCT system parameters such as: resolution, resolution degradation, distortion, sensitivity roll-off, imaging depth, scan linearity. The artefacts were characterised using an optical microscope and a swept source OCT system. Currently, quantitative methods are under development that uses these phantoms to access the performance matrices of OCT systems.

\section{ACKNOWLEDGEMENTS}

G Lee and J Rasakanthan wishes to acknowledge the funding for this research provided by the Engineering and Physical Sciences Research Council (EPSRC). In addition, J Rasakanthan gratefully acknowledges the UK Government's National Measurement Office and Sensors Knowledge Transfer for co-funding CASE Ph.D.

\section{REFERENCES}

[1] D. Huang, E. Swanson, C. Lin et al., "Optical coherence tomography," Science, 254(5035), 1178-1181 (1991).

[2] A. F. Fercher, C. K. Hitzenberger, G. Kamp et al., "Measurement of intraocular distances by backscattering spectral interferometry," Optics Communications, 117(1-2), 43-48 (1995).

[3] P. H. Tomlins, R. A. Ferguson, C. Hart et al., "Point-spread function phantoms for optical coherence tomography. ," NPL Report OP 2, 1754-2944 (2009).

[4] A. Agrawal, T. J. Pfefer, N. Gilani et al., "Three-dimensional characterization of optical coherence tomography point spread functions with a nanoparticle-embedded phantom," Opt. Lett., 35(13), 2269-2271 (2010).

[5] P. D. Woolliams, and P. H. Tomlins, "Contrast phantoms for optical coherence tomography." 7567, 756704-4.

[6] P. D. Woolliams, and P. H. Tomlins, "The modulation transfer function of an optical coherence tomography imaging system in turbid media," Physics in Medicine and Biology, 56(9), 2855 (2011).

[7] P. H. Tomlins, G. N. Smith, P. D. Woolliams et al., "Femtosecond laser micro-inscription of optical coherence tomography resolution test artifacts," Biomed. Opt. Express, 2(5), 1319-1327 (2011).

[8] J. Rasakanthan, G. C. B. Lee, P. D. Woolliams et al., "Parametric study of femtosecond inscription of microstructures for OCT artefact fabrication." 8213, 82133N-11.

[9] A. Curatolo, B. F. Kennedy, and D. D. Sampson, "Structured three-dimensional optical phantom for optical coherence tomography," Opt. Express, 19(20), 19480-19485 (2011).

[10] R. R. Gattass, and E. Mazur, "Femtosecond laser micromachining in transparent materials," Nature Photonics, 2(4), 219-225 (2008).

[11] H. O. Jeschke, M. E. Garcia, and K. H. Bennemann, "Microscopic analysis of the laser-induced femtosecond graphitization of diamond," Physical Review B, 60(6), R3701-R3704 (1999).

[12] W. Kautek, and J. Krueger, "Femtosecond pulse laser ablation of metallic, semiconducting, ceramic, and biological materials." 2207, 600-611.

[13] V. Mezentsev, M. Dubov, A. Martinez et al., "Micro-fabrication of advanced photonic devices by means of direct point-by-point femtosecond inscription in silica." 6107, 61070C-12.

[14] P. P. Pronko, S. K. Dutta, J. Squier et al., "Machining of sub-micron holes using a femtosecond laser at 800 nm," Optics Communications, 114(1-2), 106-110 (1995).

[15] S. Ortiz, D. Siedlecki, I. Grulkowski et al., "Optical distortion correction in Optical Coherence Tomography for quantitative ocular anterior segment by three-dimensional imaging," Opt. Express, 18(3), 2782-2796 (2010).

[16] P. Adrian, C. Ismini, P. Lucian et al., "Correction of distortions in optical coherence tomography imaging of the eye," Physics in Medicine and Biology, 49(7), 1277 (2004). 